\title{
Belphégor
}

Littérature populaire et culture médiatique

11-1 | 2013

Fantômas dans le siècle

\section{Pino Boero, Walter Forchesato, Felice Pozzo, Il Corsaro Nero; Nel mondo di Emilio Salgari}

Milano, Franco Angeli, 2011, p. 148 ISBN 9788856838183

\section{Fabiana Dimpflmeier}

\section{OpenEdition}

\section{Journals}

Edizione digitale

URL: http://journals.openedition.org/belphegor/183

DOI: 10.4000/belphegor.183

ISSN: 1499-7185

Editore

LPCM

Notizia bibliografica digitale

Fabiana Dimpflmeier, «Pino Boero, Walter Forchesato, Felice Pozzo, /l Corsaro Nero; Nel mondo di Emilio Salgari », Belphégor [En ligne], 11-1 | 2013, mis en ligne le 22 juin 2013, consulté le 22 septembre 2020. URL : http://journals.openedition.org/belphegor/183 ; DOI : https://doi.org/10.4000/belphegor. 183

Questo documento è stato generato automaticamente il 22 settembre 2020.

\section{(c) (i) (9)}

Belphégor est mis à disposition selon les termes de la Licence Creative Commons Attribution - Pas d'Utilisation Commerciale - Pas de Modification 4.0 International. 


\section{Pino Boero, Walter Forchesato, Felice Pozzo, Il Corsaro Nero; Nel} mondo di Emilio Salgari

Milano, Franco Angeli, 2011, p. 148 ISBN 9788856838183

Fabiana Dimpflmeier

NOTIZIA

Pino Boero, Walter Forchesato, Felice Pozzo. Il Corsaro Nero. Nel mondo di Emilio Salgari, Milano, Franco Angeli, 2011, p. 148 ISBN 9788856838183 
1 Nella collana Linee della Franco Angeli non poteva mancare, nell'anno dell'anniversario della morte di Emilio Salgari, una pubblicazione che sottolineasse l'importanza e allo stesso tempo l'attualità dello scrittore veronese attraverso l'opera che forse più di tutte ha segnato l'immaginario fantasmatico di generazioni di adolescenti e non: Il Corsaro Nero.

2 Il volume, che raccoglie tre saggi che ritornano a scavare sulla figura e le avventure del Conte Emilio di Ventimiglia come scritte dal suo omonimo padre e illustrate con i pennelli a partire da Pipein Gamba, si colloca perfettamente nell'ambito di ricerca e rielaborazione che si propone di affrontare la collana, dal sottotitolo "Laboratorio Infanzia

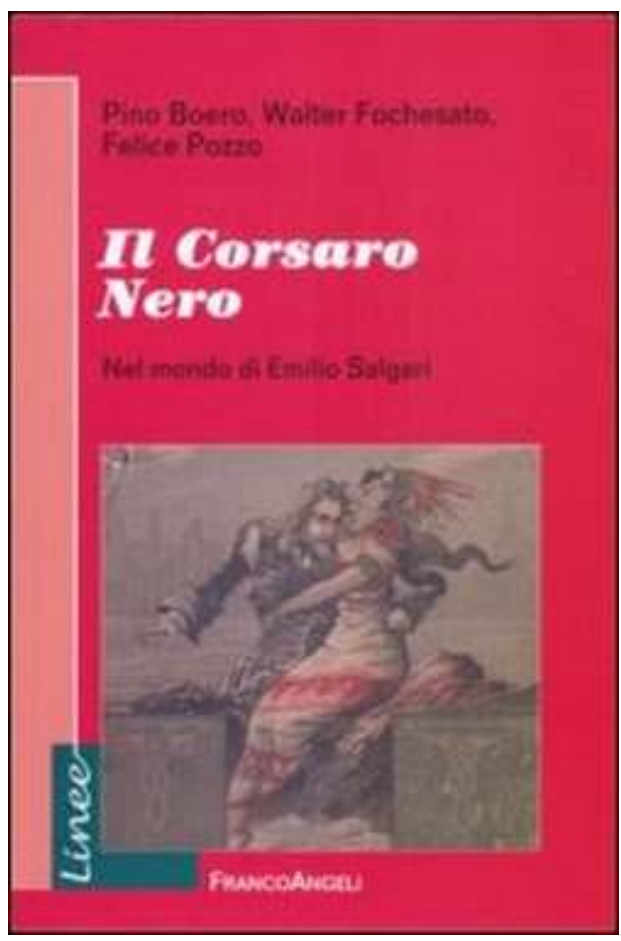
Narratività Educazione... E altro ancora", segnalando snodi inediti del romanzo e indagando particolari gustosi e densi attraverso l'analisi delle molteplici forme artistiche ed espressive utilizzate.

3 Il termine Laboratorio, in particolare, ben si collega alla 'cassetta misteriosa' di Roberto della Bohème Italiana e al tavolino di Salgari come volontà di costruire un ponte fra un passato salgariano e un presente dalla rinnovata progettualità didattica, in una rielaborazione critica dei materiali e attraverso un approfondimento in chiave innovativa di quello che è oramai acquisito come un classico della letteratura dell'infanzia, nonostante gli esordi e la fruizione continua da parte di un pubblico 'adulto' - come dimostrato nel recente adattamento di Francesca Manieri dal 7 al 12 febbraio 2012 al Teatro Argentina di Roma.

4 E proprio su cosa sia accaduto nell'officina segreta di Salgari, sul suo tavolino oscillante e a gambe mobili, nel pieno della sua fucina creativa, è ciò che indaga Felice Pozzo nel suo articolo "Il tempo perduto dei corsari gentiluomini". Il decano degli studiosi salgariani si destreggia fra i più reconditi e sottili aspetti del romanzo, conducendo il lettore paragrafo dopo paragrafo alla scoperta dei minuti particolari che solo una ripetuta, appassionata ed accorta lettura può svelare a chi non è abituato a fare attenzione alle parole e al suono che rimandano nell'eco di una cassa armonica ben più ampia di quella della singola opera, e che si lega al contesto storico, artistico e culturale.

5 Così passiamo dai possibili richiami alla storia reale - la guerra ispano americana a Cuba - a quelli della famiglia Reale dei Savoia, dagli scambi di scherma, alla bandiera di Verne, al Corsaro rosso di Cooper, al Il Conte di Montecristo di Dumas, alla musica contemporanea per arrivare alla versione 'erotica' di Honorata e allo spunto sull'illusionismo, alla bellezza che trionfa sui selvaggi nel Guarany e alle personificazioni allegoriche delle moralities, vere chicche per lettori voraci e studiosi alla ricerca di piste inedite... 
6 Felice Pozzo, con la sua veloce carrellata, ha la brillante capacità di mostrarci Il Corsaro nero come un prisma capace di proiettare ad ogni ulteriore e successiva lettura nuove sfumature e ombre. Ma sembra trasparire dalle sue parole la ferrea volontà di mostrare ancora una volta e pedissequamente quanto sia 'egregiamente strutturata' l'officina creativa di Salgari, quando forse oramai, come anche lui in fine riconosce, non ve n'è più bisogno.

7 Partendo proprio dalla necessità di un'analisi diversa, Pino Boero imposta il secondo saggio ("Le rotte dell'avventura") all'insegna della sottrazione, intesa come un invito e un ritorno a far parlare il testo in sé e a sondarne i contenuti - 'la grammatica dell'immaginario' - seguendo i suggerimenti offerti dall'antropologia nell'analisi della contrapposizione fra cultura egemone e cultura subalterna, intesa in senso allargato come cultura popolare, contadina, infantile e femminile.

8 Una cultura dell'infanzia, perché di questa stiamo parlando, che "non diversamente dalla cultura contadina nei lunghi secoli della sua storia [è] una cultura silenziosa, priva di un proprio autonomo linguaggio, costretta inevitabilmente a parlare il linguaggio della cultura dominante, e a cercare nei processi retorici della metaforizzazione e della deformazione espressiva i propri strumenti di identificazione e di sfogo" (Remo Cesaroni cit. nel testo).

9 Il paragone è interessante se si riflette sul fatto che esiste letteratura per l'infanzia ma non letteratura scritta dall'infanzia e che quindi i processi retorici e la deformazione espressiva di cui parla Cesaroni non possono che essere considerati ex post (insomma, usciti dalla penna di scrittori non più bambini) o come il frutto del successo presso il pubblico, i cui gusti in fatto di lettura fanno da cartina di tornasole delle scelte dello scrittore e lo aiutano a crescere in un percorso elicoidale.

Da questo punto di vista mi sembra che la domanda di fondo che guida i 'sondaggi' sui testi e suggerita in più punti sia: "quali sono le immagini, le parole, gli oggetti, gli atteggiamenti e gli usi descritti nel testo che hanno accomunato i lettori del Corsaro Nero e che hanno reso la sua fruizione possibile negli anni ?". Una domanda che, per contrasto, potrebbe aiutare a capire proprio perché 'Salgari stia oggi perdendo i giovani', forse non più 'incardinato attraverso le parole nell'immaginario delle nuove generazioni".

11 Ciò che accomuna la cultura dell'infanzia e la cultura popolare è allora l'appartenenza ad una categoria di 'esclusi' - rispetto alle letture castranti e calate dall'alto della didattica scolastica dell'epoca (e non solo) -, e anche, e soprattutto, una comunione di orizzonti pratici, il fatto cioè che la maggior parte dei bambini che leggeva Salgari faceva parte proprio di quel marasma di marmocchi, poveri e affamati, i cui padri e madri coltivavano i campi, emigravano in città o al di là dell'oceano, esemplari viventi di quell'etnografia italiana che fra la fine dell'Ottocento e i primi del Novecento insigni antropologi si affannavano a raccogliere per documentare i veri costumi dell'Itala gente dalle molte vite' e che, come suggerisce Boero, una rilettura del Giannettino di Collodi difficilmente potrebbe aiutare a ricordare.

12 Lo studioso riesce ad individuare la domesticità dell'Italia più povera sotto la patina di esotismo dell'opera di Salgari, oltre ad alcuni dei molteplici aspetti che la hanno resa memorabile, conturbante e appetibile alle generazioni. Da una parte i lettori si rispecchiano nel romanzo perché percepiscono una realtà di fondo condivisibile nelle pulsioni più elementari, nelle parole, nella corporeità metaforizzata, nei turbamenti... 
Dall'altra sono accomunati dalla forza vivida e immaginifica dell'avventura come forma di fuga da quello stesso quotidiano che permette loro di entrare a far parte del romanzo.

13 L'esorcizzazione della quotidianità che Salgari offre in risposta 'al male di vivere' italiano attraverso la tecnica dello straniamento, l'innesto dell'esotico sul domestico, permette infatti, per una trasposizione interna di equilibri, di operare una sorta di 'esoticizzazione' del nostrano attraverso una presa di distanza da quello che Giuseppe Cocchiara ha definito il 'selvaggio interno'.

Terzo ed ultimo saggio dal titolo divertente e quanto mai azzeccato "Avventure di pennini e pennelli", è quello di Walter Forchesato in cui, partendo da Pipein Gamba, Alberto dalla Valle e Gennaro Amato, l'autore mostra chiaramente quanto il legame che pur vincola gli illustratori al canone descrittivo salgariano - il rapporto inevitabile tra scrittura e immagine - riesca a trasformarsi in uno scambio nutriente fra originalità e autonomia di mano dei disegnatori e creazione artistica della figura del Corsaro, che ne assorbe vita e stile dando vita a un preciso e denso parametro figurativo per le generazioni successive: "un signore di Ventimiglia che passa indenne fra foreste e assalti, colpi di mare e assedi, inseguimenti e arrembaggi senza che mai il suo vestito si strappi o perda la piega, senza che gli stivali si sciupino in punta o il cappello diventi flaccido per l'acqua di mare o l'umidità mefitica delle paludi" (p. 116). Un canone rappresentativo a cui queste cercheranno di mantenersi fedeli, con risultati tuttavia altalenanti.

Dalle derivazioni teatrali delle tavole di Pipein Gamba fino ai successi televisivi e più moderni dei fumetti avviene infatti una rielaborazione continua, un ri-masticamento di colori e di tratti più o meno riuscito, ma che ben testimonia, financo e forse soprattutto nella parodia, dell'affetto dei lettori di Salgari e della sua presenza nel loro immaginario: una dimensione che appare policroma, stratificata, a volte pastosa, a volte leggera.

La ricchezza di informazioni e di nomi, il rincorrersi di vicende e angoli ancora inesplorati nella storia dell'illustrazione che riccamente ci restituisce Forchesato, corredati da squisitezze da vero amatore, fanno del saggio un canovaccio degno di un'opera ben più matura, quasi fosse la sua 'cassetta misteriosa', a noi preziosamente svelata in anteprima.

17 Tutti e tre i saggi, infine, richiamano alla necessità di un'analisi storica e culturale del cambiamento avvenuto nel corso degli ultimi anni nel processo di rispecchiamento del pubblico giovanile, fagocitato da una nuova e preponderante dimensione visiva e sempre più lontano dalle dinamiche valoriali salgariane, che però sembriamo un po' tutti solo rispettare e rimpiangere, evitando di inoltrarci nella nostra quotidianità e di confrontarci dal vivo con i nostri figli. 


\section{AUTORI}

\section{FABIANA DIMPFLMEIER}

Università di Roma 'La Sapienza' 